\title{
Multiparametric exercise stress cardiovascular magnetic resonance in the diagnosis of coronary artery disease: the EMPIRE trial
}

Thu-Thao Le ${ }^{1,2^{*}}$, Briana W. Y. Ang ${ }^{1}$, Jennifer A. Bryant ${ }^{1,2}$, Chee Yang Chin ${ }^{1,2,3}$, Khung Keong Yeo ${ }^{1,2,3}$, Philip E. H. Wong ${ }^{1,2,3}$, Kay Woon Ho ${ }^{1,2,3}$, Jack W. C. Tan ${ }^{1,2,3}$, Phong Teck Lee ${ }^{1,2,3}$, Calvin W. L. Chin 1,2,3† and Stuart A. Cook $1,2,3,4+$

\begin{abstract}
Background: Stress cardiovascular magnetic resonance (CMR) offers assessment of ventricular function, myocardial perfusion and viability in a single examination to detect coronary artery disease (CAD).

We developed an in-scanner exercise stress CMR (ExCMR) protocol using supine cycle ergometer and aimed to examine the diagnostic value of a multiparametric approach in patients with suspected CAD, compared with invasive fractional flow reserve (FFR) as the reference gold standard.

Methods: In this single-centre prospective study, patients who had symptoms of angina and at least one cardiovascular disease risk factor underwent both ExCMR and invasive angiography with FFR. Rest-based left ventricular function (ejection fraction, regional wall motion abnormalities), tissue characteristics and exercise stress-derived (perfusion defects, inducible regional wall motion abnormalities and peak exercise cardiac index percentile-rank) CMR parameters were evaluated in the study.

Results: In the 60 recruited patients with intermediate CAD risk, 50\% had haemodynamically significant CAD based on FFR. Of all the CMR parameters assessed, the late gadolinium enhancement, stress-inducible regional wall motion abnormalities, perfusion defects and peak exercise cardiac index percentile-rank were independently associated with FFR-positive CAD. Indeed, this multiparametric approach offered the highest incremental diagnostic value compared to a clinical risk model ( $X^{2}$ for the diagnosis of FFR-positive increased from 7.6 to $\left.55.9 ; P<0.001\right)$ and excellent performance [c-statistic area under the curve 0.97 (95\% Cl: 0.94-1.00)] in discriminating between FFR-normal and FFR-positive patients.
\end{abstract}

Conclusion: The study demonstrates the clinical potential of using in-scanner multiparametric ExCMR to accurately diagnose CAD.

Trial registration: ClinicalTrials.gov, NCT03217227, Registered 11 July 2017-Retrospectively registered, https://clinicaltr ials.gov/ct2/show/NCT03217227?id=NCT03217227\&draw=2\&rank=1\&load=cart

\footnotetext{
*Correspondence: le.thu.thao@nhcs.com.sg

${ }^{\dagger}$ Calvin W. L. Chin and Stuart A. Cook co-senior authors

${ }^{1}$ National Heart Research Institute Singapore, National Heart Centre

Singapore, 5 Hospital Drive, Singapore 169609, Singapore

Full list of author information is available at the end of the article
}

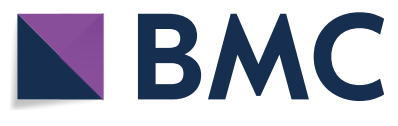

(c) The Author(s) 2021. Open Access This article is licensed under a Creative Commons Attribution 4.0 International License, which permits use, sharing, adaptation, distribution and reproduction in any medium or format, as long as you give appropriate credit to the original author(s) and the source, provide a link to the Creative Commons licence, and indicate if changes were made. The images or other third party material in this article are included in the article's Creative Commons licence, unless indicated otherwise in a credit line to the material. If material is not included in the article's Creative Commons licence and your intended use is not permitted by statutory regulation or exceeds the permitted use, you will need to obtain permission directly from the copyright holder. To view a copy of this licence, visit http://creativecommons.org/licenses/by/4.0/. The Creative Commons Public Domain Dedication waiver (http://creativeco mmons.org/publicdomain/zero/1.0/) applies to the data made available in this article, unless otherwise stated in a credit line to the data. 
Keywords: Exercise stress, Supine cycle ergometer, Coronary artery disease, Cardiovascular magnetic resonance, Fractional flow reserve

\section{Introduction}

Stress cardiovascular magnetic resonance (CMR) has evolved as a highly accurate non-invasive diagnostic [13] and prognostic [4-6] test that provides cost-effective imaging-based strategy to guide coronary revascularization $[7,8]$ in patients with stable coronary artery disease (CAD). Advantages of CMR include its excellent spatial resolution, independence of acoustic windows, free from ionising radiation and multi-parametric assessment of ventricular function, myocardial perfusion and viability in a single examination.

Exercise stress tests are more physiological in replicating the symptoms and haemodynamic changes compared to pharmacological-induced stress, with less adverse events and better tolerated [9-11]. However, due to limited availability of CMR-conditional stress equipment that precludes physiological-induced stress, conventional stress CMR typically refers to pharmacological-induced stress perfusion CMR [12]. Only recently has exercise treadmill CMR made inroads into the diagnosis of CAD, with a multi-centre trial demonstrated excellent agreement between exercise treadmill CMR and coronary angiography [13].

We have developed an exercise stress CMR (ExCMR) protocol using an in-scanner cycle ergometer [14] and established normal exercise capacities (peak exercise cardiac index percentile-rank; Peak $_{\mathrm{CI}}$ ) in the local healthy population [15]. The in-scanner stress protocol allows imaging to be done at every stage of exercise. Peak $\mathrm{CI}_{\mathrm{CI}}$ has been validated against the maximal oxygen uptake $\left(\mathrm{VO}_{2} \mathrm{max}\right)$ of cardiopulmonary stress test [14] and a low Peak $_{\mathrm{CI}}(<35$ th percentile for age and sex) discriminates between pathological and physiological cardiac remodelling [15]. Extending this work, we aim to examine the diagnostic value of ExCMR in patients with suspected CAD, compared with the gold standard invasive fractional flow reserve (FFR) to define significant CAD. We hypothesized that a multiparametric approach of CMR parameters would have a high diagnostic accuracy for CAD.

\section{Methods}

\section{Study design and patient population}

The EMPIRE trial (Evaluating Myocardial Ischaemia in Chest Pain Using Exercise CMR; ClinicalTrials.gov identifier: NCT03217227) is a single-centre prospective study. The patients who had symptoms of angina (Canadian Class Symptoms CCS class II or III [16]) and at least one cardiovascular disease risk factor (diabetes, hypertension, dyslipidaemia, smoking, or family history of CAD). Those patients who were recommended for invasive coronary angiograms by their cardiologists and agreed to participate in the study were recruited. Exclusion criteria were previous coronary artery interventions (coronary artery bypass grafting; and/or percutaneous coronary intervention), acute coronary syndromes (unstable angina, non-ST elevation myocardial infarction and ST elevation myocardial infarction), physical disabilities that would preclude exercise testing, contraindications to contrastenhanced CMR, and inability to tolerate adenosine (for FFR assessment).

The study was conducted in accordance to the Declaration of Helsinki and approved by the Singhealth Centralised Institutional Review Board. Written informed consent was obtained from all participants prior to CMR and invasive coronary angiogram.

\section{Exercise CMR protocol}

Patients taking beta blockers were advised to stop taking them 2 days before stress test. Baseline breath-hold CMR cine images and native T1 maps were acquired in all patients before initiating exercise stress (60-channel phased-array coil, MAGNETOM Aera 1.5T, Siemens Healthineers, Erlangen, Germany). ExCMR was performed with a programmable supine ergometer (Lode BV, Groningen, the Netherlands) fitted onto the CMR scanner table. Patients were asked to cycle at the initial workload of $25 \mathrm{~W}$, with cadence maintained at least $70 \mathrm{rpm}$ for $1 \mathrm{~min}$. Workload was increased by $25 \mathrm{~W}$ every minute until exhaustion or presence of symptoms (dyspnoea or chest pain). Free-breathing imaging was done at the end of every stage during a brief period of stopping exercise to minimize motion and electrocardiogram (ECG) triggering related artefacts. After free-breathing image acquisition at peak exercise, patients were asked to continue cycling for another minute to maintain the maximal heart rate. On exercise termination, freebreathing first-pass stress perfusion was performed with the injection of $0.075 \mathrm{mmol} / \mathrm{kg}$ gadolinium contrast agent (Gadovist; Bayer Pharma AG, Berline, Germany). Rest perfusion imaging was acquired $10 \mathrm{~min}$ after stress, with a further injection of $0.075 \mathrm{mmol} / \mathrm{kg}$ gadolinium, at the same slice locations. Late gadolinium enhancement (LGE) imaging was performed $10 \mathrm{~min}$ after the 2nd dose of contrast injection, followed by post-contrast T1 maps at $15 \mathrm{~min}$ after contrast. The higher gadolinium dosage 
of $0.075 \mathrm{mmol} / \mathrm{kg}$ was given to maintain the contrastto-noise ratio for both perfusion and LGE imaging. The total duration of the ExCMR protocol was $60 \mathrm{~min}$. Blood pressure was monitored at every stage of exercise, during stress and rest perfusion imaging and recovery. A fivepoint score was used to assess patient's experience at the end of ExCMR: $1=$ would not do it again, $2=$ somewhat uncomfortable, $3=$ neutral, $4=$ somewhat comfortable, $5=$ highly satisfied.

The imaging sequences and parameters were as follows and illustrated in Fig. 1:

\section{Breath-hold cines}

Balanced steady-state free precession (bSSFP) of long axis (2-, 4-chamber and sagittal left ventricular (LV) outflow tract) and short axis cines extending from base to apex $(8 \mathrm{~mm}$ thick and $2 \mathrm{~mm}$ gap; TE $=1.2 \mathrm{~ms}$; TR $=3 \mathrm{~ms}$; FOV 280-320 mm; 13 segments per phase; acquired matrix size $205 \times 256$ pixels; acceleration factor 2 ; acquired voxel size $1.6 \times 1.3 \times 8 \mathrm{~mm}$; temporal resolution $39 \mathrm{~ms} ; 30$ phases per cardiac cycle).

\section{Real-time free-breathing cines}

bSSFP of short axis cines: prospective ECG-gated free-breathing image acquisition of $10-13$ short axis slices for at least 2 cardiac cycles, extending from base to apex, at every stage of exercise $(8 \mathrm{~mm}$ thick and $2 \mathrm{~mm}$ gap; $\mathrm{TE}=0.99 \mathrm{~ms} ; \mathrm{TR}=2.3 \mathrm{~ms}$; flip angle $56^{\circ}$; FOV $230 \times 300 \mathrm{~mm}$; acquired matrix size $68 \times 128$ pixels; acceleration factor 4; acquired voxel size $3.4 \times 2.3 \times 8 \mathrm{~mm}$; temporal resolution $39 \mathrm{~ms} ; 10$ to 30 phases per cycle depending on heart rate).

\section{Stress and rest perfusion}

Free-breathing bSSFP sequence of 4 short axis slices over 2 concatenations (slice thickness $8 \mathrm{~mm}$; TE $=0.98 \mathrm{~ms}$; $\mathrm{TR}=2.3 \mathrm{~ms}$; flip angle $50^{\circ}$; FOV $263 \times 350 \mathrm{~mm}$; acquired matrix size $98 \times 160$ pixels; acceleration factor 2 ; acquired voxel size $2.6 \times 2.1 \times 8 \mathrm{~mm}$ ). Images were motion corrected by the unsupervised inline process implemented within the Image Calculation Environment of Siemens MR system [17] (Additional file 1).

\section{Late-gadolinium enhancement}

Phase-sensitive inversion recovery (PSIR) magnitudereconstructed images of 3 standard long-axis and shortaxis stack from base to apex $(8 \mathrm{~mm}$ thick and $2 \mathrm{~mm}$ gap; $\mathrm{TE}=1.19 \mathrm{~ms} ; \mathrm{FOV} 270 \times 360 \mathrm{~mm}$; acquired matrix size $144 \times 256$ pixels; acceleration factor 2 ; acquired voxel size $1.9 \times 1.4 \times 8 \mathrm{~mm}$; inversion time adjusted individually to achieve appropriate nulling of the myocardium).

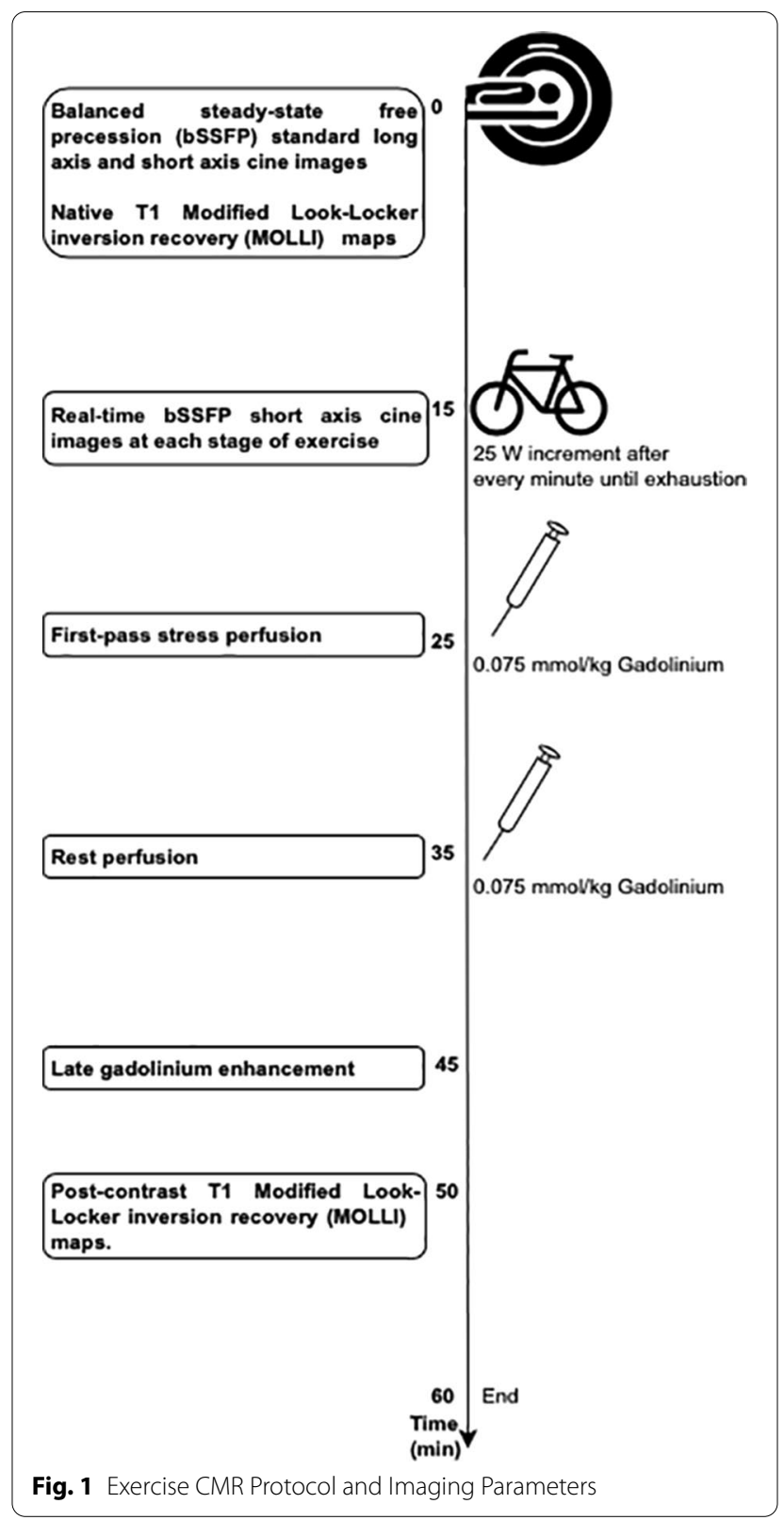

Myocardial native and post-contrast $T 1$ maps

Modified Look-Locker inversion recovery (MOLLI) sequence with 5(3)3 scheme at basal and mid-ventricular levels $(8 \mathrm{~mm}$ thick; $\mathrm{TE}=1.12 \mathrm{~ms}$; FOV $270 \times 360 \mathrm{~mm}$; acquired matrix size $144 \times 256$ pixels; acceleration factor 2 ; flip angle $35^{\circ}$; acquired voxel size 
$1.9 \times 1.4 \times 8 \mathrm{~mm}$; minimum $\mathrm{TI}=100 \mathrm{~ms}$ and $80 \mathrm{~ms}$ increment; inline motion correction).

\section{CMR image analysis}

LV mass and cardiac volumes at baseline (breathhold short axis cine images) and each exercise stage (real-time short axis cine images) were measured in all patients using standardized protocols at our NHRIS CMR Core Laboratory (cvi42, Circle Cardiovascular Imaging, Calgary, Alberta, Canada) [14]. Exercise-related CMR measures assessed in this study included relative change in exercise LV ejection fraction (LVEF), relative change in exercise indexed stroke volume (SVI), relative change in exercise cardiac index, and Peak $_{\mathrm{CI}}$ expressed as age- and sex-specific percentiles according to the reference ranges previously established [15]. LGE patterns (ischaemic versus nonischaemic) and the extent of infarction were visually assessed based on the 16-segment model. Ischaemic LGE pattern was defined as enhancement in a vascular distribution (subendocardial to transmural) [18]. LGE $>50 \%$ of myocardial wall thickness was considered non-viable [19]. Native T1 was derived by manually contouring the endocardium and epicardium of the native T1 maps at basal and mid-ventricular levels using standardized protocol [20], excluding infarcted regions as recommended [21]. Ischaemia on T1 maps are regions corresponding to reversible perfusion defects and confirmed by angiographic evidence of significant stenosis [22]. Extracellular volume (ECV) was calculated from native and post-contrast $\mathrm{T} 1$ values as:( 1 - haematocrit $) \times\left(\Delta \mathrm{R} 1_{\text {myocardium }}-\Delta \mathrm{R} 1_{\text {blood }}\right)$, where $\mathrm{R} 1=1 / \mathrm{T} 1$. Haematocrit was taken on the day of CMR scan.

Qualitative assessment of inducible regional wall motion abnormalities, perfusion defects and LGE was performed according to contemporary recommendations [23], by two independent CMR physicians prior to invasive coronary angiography. Any discrepancies were resolved by consensus before the coronary angiography was performed. The qualitative components of the ExCMR was recorded as: presence/absence of inducible regional wall motion abnormalities (RWMA), presence/ absence of perfusion defects. Presence of hypoperfusion at both stress and rest without corresponding hyperenhancement region on LGE images was considered as artefact. In addition, 9 out of 60 cases (15\%) were evaluated by 3 experienced CMR readers to assess the interobserver reproducibility. All readers were blinded to CMR and FFR reports.

\section{Invasive coronary angiography and assessment} of fractional flow reserve

All patients underwent invasive coronary angiography within 1 month after ExCMR by interventionalists who were briefed to follow the angiography/FFR study protocol. FFR was assessed using standard procedure [24] (PressureWire $^{\mathrm{TM}} \mathrm{X}$ Guidewire, Abbott Laboratories, Chicago, Illinois, USA), in all coronary arteries with a calibre of $2.5 \mathrm{~mm}$ or more and a stenosis severity of $40 \%$ or more if technically feasible, as determined by the interventionalist. Maximal hyperaemia was induced by intravenous adenosine infusion at a rate of $180 \mu \mathrm{g} / \mathrm{kg} / \mathrm{min}$ for at least 2 min for FFR calculation. An FFR value of $\leq 0.8$ or total occlusion was defined as FFR-positive [25]. Total occluded coronary artery was assigned an FFR value of 0.5. The interventionalists were blinded to the findings of the CMR scans before the procedure.

\section{Statistical analysis}

Distribution of continuous variables was assessed using the Shapiro-Wilk test. Data were presented in either mean \pm standard deviation or median [inter-quartile range], as appropriate. Depending on the normality of the distribution, parametric Student's t test and 1-way ANOVA or the nonparametric Mann-Whitney $U$ test and Kruskal-Wallis test were used to compare groups of continuous data. Categorical data were compared using the $\mathrm{X}^{2}$ test. A two-sided P-value $<0.05$ was considered as statistically significant. Cohen's kappa ( $\mathrm{k}$ ) was used to assess agreement between pairs of readers. The agreement grading based on $\mathrm{k}$ values were: poor $(0-0.02)$, fair $(0.21-0.40)$, moderate $(0.41-0.60)$, substantial $(0.61-$ $0.80)$ and nearly perfect $(0.81-1.00)$.

Determinants associated with FFR-positive were assessed using univariate and backward selection multivariable Firth logistic regression to handle 0 cell frequency. Clinically relevant variables that demonstrated independent association $(P<0.10)$ with significant CAD defined by FFR were retained in the final model [26]. The incremental diagnostic value of the multiparametric model, consisting of CMR variables that were retained from the multivariable analysis, over a clinical model consisting of age, sex and coronary risk factors (diabetes, hypertension, dyslipidaemia and smoking history) and conventionally used CMR parameters for assessment of CAD (RWMA, perfusion defects and LGE) was assessed using a change in the global $X^{2}$. The diagnostic performance of this multiparametric approach was tested using the c-statistics for discrimination (area under the receiver operating characteristic curve; AUC).

Statistical analyses were performed using GraphPad Prism (version 8.3.0, GraphPad Software, Inc., San Diego, 
California, USA), SPSS (version 26, Statistical Package for the Social Sciences, International Business Machines, Inc., Armonk, New York, USA) and R package (version 3.5.3, R Foundation for Statistical Computing, Vienna, Austria).

\section{Results}

\section{Baseline characteristics of the study participants}

A total of 60 patients were recruited in the study between May 2017 and December 2019 (Additional file 1). All patients completed both ExCMR and invasive FFR assessment. The median time between ExCMR and FFR was $7[6,8]$ days. Of the 60 patients, $30(50 \%)$ were FFRpositive: 14 (47\%) one-vessel disease (1VD), 9 (30\%) twovessel disease (2VD) and 7 (23\%) three-vessel disease (3VD). Patients had intermediate CAD pre-test probability based on the CAD Consortium Clinical Pre-test Probability Score (mean $38 \pm 22 \%$ ). Patients who were FFR-positive had higher pre-test probability compared to FFR-normal ( $45 \%$ versus $30 \%, P=0.005)$. Non-ischemic LGE was present in more patients who were FFR-normal compared to FFR-positive (17 versus $7 \%$ respectively; $P<0.001)$. Of note, native T1 and ECV values were similar between the groups $(P>0.05$; Table 1); and there was no significant difference in native T1 between ischaemic and non-ischaemic regions $(1014 \pm 39 \mathrm{~ms}$ versus $1021 \pm 30 \mathrm{~ms} ; P=0.334$ ).

\section{Cardiac Response to Exercise CMR}

The ExCMR scan took $62[56,70]$ mins, with the stress component completed within $10[8,13]$ mins. Exercise stress was prematurely terminated in 4 (7\%) patients due to symptoms. Peak exercise heart rate (HR) achieved was $122 \pm 19 \mathrm{bpm}$, corresponding to $76 \pm 11 \%$ of the age-predicted maximal heart rate (APMHR). Peak rate-pressure product was $21,300 \pm 5,020 \mathrm{mmHg} / \mathrm{min}$. Patients in both groups achieved similar peak HR, peak blood pressure and peak rate-pressure product (Table 1). ExCMR protocol was well-tolerated by all patients (median satisfaction score of $4[3,4])$. The agreement between readers was substantial for perfusion $(\kappa=0.68)$ and nearly perfect for RWMA ( $\kappa=0.85)$ and LGE $(\kappa=1.00)$.

Of all the ExCMR quantitative parameters, only Peak $\mathrm{CI}_{\mathrm{CI}}$ correlated positively with FFR $(\mathrm{r}=0.401 ; \mathrm{P}=0.002)$. No FFR-positive patients had a Peak $_{\mathrm{CI}}$ above $35^{\text {th }}$ percentile specific for age and sex. Moreover, Peak $_{\mathrm{CI}}$ decreased significantly with more severe CAD (1VD: $8^{\text {th }}[3,9]$ percentile; $2 V D: 4^{\text {th }}[2,10]$ percentile; $3 V D: 2^{\text {nd }}[0,3]$ percentile; $\mathrm{P}=0.045$ ). Perhaps not surprisingly, exercise stress-induced RWMA, perfusion defects and myocardial infarction on LGE were markedly more prevalent in FFR-positive patients (Table 1). Conversely, there was no correlation between FFR values and segments of perfusion defect $(\rho=-0.015 ; \mathrm{P}=0.0937)$ or infarction on LGE $(\rho=0.07 ; \mathrm{P}=0.807)$. The other ExCMR measures (change in indexed LV stroke volume (SV), LVEF and cardiac index) did not differ significantly between those patients with FFR-positive and FFR-normal (Table 1).

\section{Diagnostic characteristics of multiparametric exercise stress CMR parameters}

Of all the CMR parameters assessed, only LGE, stressinducible RWMA, perfusion defects and Peak $\mathrm{CI}_{\mathrm{I}}$ maintained an independent association on multivariate analysis with CAD defined by FFR (Table 2). Indeed, this multiparametric "4-in-1" approach (Central Illustration) demonstrated incremental diagnostic value over a clinical model. As a marker of exercise capacity, Peak $_{\mathrm{CI}}$ demonstrated incremental diagnostic value over inducible RWMA, perfusion defects and LGE (Fig. 2). The "4-in-1" model had the highest diagnostic accuracy [AUC 0.97 (95\% CI: 0.94-1.00)], although the difference was non-significant when compared to the combination of conventional stress CMR parameters (inducible RWMA and perfusion) and LGE [AUC 0.94 (95\% CI: 0.87-1.00); $P=0.136$ for the difference between AUCs]. Similar results were observed when patients were stratified to above median APMHR [0.99 (95\% CI: 0.96-1.00) and below median APMHR [0.95 (95\% CI: 0.88-1.00); $P=0.339$ for difference between AUCs] (Fig 3).

\section{Discussion}

EMPIRE is the first study to examine the potential of a multiparametric in-scanner ExCMR protocol to diagnose significant $\mathrm{CAD}$, when compared with the gold standard invasive FFR. We have demonstrated that in patients with intermediate pre-test CAD risk, the ExCMR multiparametric approach consists of LGE patterns, stress-inducible RWMA, perfusion defects, and exercise capacity offered the highest incremental diagnostic value over a clinical model and excellent diagnostic accuracy [AUC 0.97 (95\% CI: 0.94-1.00), $P<0.001$ ].

Exercise is the most physiological stress technique. In recent years, we along with others have demonstrated the increasing potential of exercise CMR [13-15, 27, 28]. Assessing regional wall motion abnormalities and perfusion defects in a single stress imaging is not routinely performed with conventional adenosine stress techniques. The current study demonstrated the feasibility of the in-scanner supine cycling exercise CMR protocol to assess both regional wall motion abnormalities and perfusion defects. Using in-scanner cycling exercise, there is minimal delay in imaging at peak stress and acquisition at every exercise stage may 
Table 1 Clinical characteristics and CMR parameters at rest and stress

\begin{tabular}{|c|c|c|c|c|}
\hline & All patients $(n=60)$ & FFR-normal $(n=30)$ & FFR-positive $(n=30)$ & $P$ Value \\
\hline \multicolumn{5}{|l|}{ Clinical characteristics } \\
\hline Age, years & $59 \pm 8$ & $57 \pm 9$ & $61 \pm 6$ & 0.095 \\
\hline Males, n (\%) & $43(72)$ & $20(67)$ & $23(77)$ & 0.399 \\
\hline $\mathrm{BMI}, \mathrm{kg} / \mathrm{m}^{2}$ & $26.1 \pm 3.7$ & $26.1 \pm 4.1$ & $26.0 \pm 3.2$ & 0.901 \\
\hline $\mathrm{BSA}, \mathrm{m}^{2}$ & $1.77 \pm 0.18$ & $1.80 \pm 0.20$ & $1.75 \pm 0.15$ & 0.342 \\
\hline Diabetes mellitus, n (\%) & $28(47)$ & $14(47)$ & $14(47)$ & 1.000 \\
\hline Hypertension, n (\%) & $49(82)$ & $23(77)$ & $26(87)$ & 0.325 \\
\hline Dyslipidaemia, n (\%) & $50(83)$ & $25(83)$ & $25(83)$ & 1.000 \\
\hline Smoking, n (\%) & $17(28)$ & $8(27)$ & $9(30)$ & 0.774 \\
\hline Family history of CAD, n (\%) & $26(43)$ & $13(43)$ & $13(43)$ & 1.000 \\
\hline Pre-test CAD consortium clinical score, $\%$ & $38 \pm 22$ & $30 \pm 20$ & $45 \pm 22$ & 0.005 \\
\hline $\begin{array}{l}\text { Medication, } n(\%) \\
\text { ACE inhibitor } \\
\text { ARB } \\
\text { Statin } \\
\text { Other lipid-lowering drug } \\
\text { Beta blocker }\end{array}$ & $\begin{array}{l}7(12) \\
22(37) \\
53(88) \\
11(18) \\
41(68)\end{array}$ & $\begin{array}{l}6(20) \\
9(30) \\
25(83) \\
3(10) \\
19(63)\end{array}$ & $\begin{array}{l}1(3) \\
13(43) \\
28(93) \\
8(27) \\
22(73)\end{array}$ & $\begin{array}{l}0.044 \\
0.284 \\
0.228 \\
0.095 \\
0.405\end{array}$ \\
\hline Systolic blood pressure, mmHg (rest) & $139 \pm 16$ & $141 \pm 14$ & $137 \pm 18$ & 0.394 \\
\hline Diastolic blood pressure, mmHg (rest) & $79 \pm 13$ & $79 \pm 13$ & $79 \pm 11$ & 0.943 \\
\hline Heart rate, bpm (rest) & $73 \pm 12$ & $74 \pm 11$ & $72 \pm 12$ & 0.473 \\
\hline Rate-pressure product, $\mathrm{mmHg} / \mathrm{min}$ (rest) & $10,106 \pm 2141$ & $10,377 \pm 2004$ & $9835 \pm 2271$ & 0.330 \\
\hline Systolic blood pressure, mmHg (stress) & $174 \pm 26$ & $175 \pm 29$ & $172 \pm 23$ & 0.650 \\
\hline Diastolic blood pressure, mmHg (stress) & $111 \pm 29$ & $115 \pm 30$ & $107 \pm 28$ & 0.319 \\
\hline Heart rate, bpm (stress) & $122 \pm 19$ & $123 \pm 19$ & $121 \pm 20$ & 0.558 \\
\hline Age-predicted maximal heart rate, $\%$ & $76 \pm 11$ & $76 \pm 10$ & $76 \pm 12$ & 0.953 \\
\hline Rate-pressure product, mmHg/min (stress) & $21,300 \pm 5017$ & $21,721 \pm 5324$ & $20,879 \pm 4743$ & 0.520 \\
\hline \multicolumn{5}{|l|}{ CMR parameters at rest } \\
\hline Indexed LV mass, $\mathrm{g} / \mathrm{m}^{2}$ & $50 \pm 12$ & $50 \pm 14$ & $49 \pm 9$ & 0.765 \\
\hline Indexed LV EDV, $\mathrm{mL} / \mathrm{m}^{2}$ & $76 \pm 15$ & $76 \pm 15$ & $76 \pm 15$ & 0.892 \\
\hline Indexed LV ESV, mL/m² & $33 \pm 12$ & $31 \pm 10$ & $35 \pm 13$ & 0.201 \\
\hline Indexed LVSV, mL/m² & $43 \pm 8$ & $44 \pm 9$ & $41 \pm 8$ & 0.102 \\
\hline LVEF, \% & $57 \pm 9$ & $59 \pm 7$ & $55 \pm 9$ & 0.039 \\
\hline Indexed RV EDV, $\mathrm{mL} / \mathrm{m}^{2}$ & $77 \pm 14$ & $78 \pm 17$ & $75 \pm 11$ & 0.432 \\
\hline Indexed RV ESV, mL/m² & $34 \pm 10$ & $34 \pm 10$ & $34 \pm 9$ & 0.906 \\
\hline Indexed RV SV, mL/m² & $42 \pm 8$ & $44 \pm 9$ & $41 \pm 8$ & 0.110 \\
\hline RVEF, \% & $56 \pm 8$ & $57 \pm 7$ & $55 \pm 9$ & 0.228 \\
\hline Cardiac index, $\mathrm{L} / \mathrm{min} / \mathrm{m}^{2}$ & $3.1 \pm 0.7$ & $3.2 \pm 0.7$ & $3.0 \pm 0.7$ & 0.212 \\
\hline \multicolumn{5}{|l|}{ CMR tissue characteristics } \\
\hline $\begin{array}{l}\text { Late gadolinium enhancement } \\
\text { Ischaemic, } \mathrm{n}(\%) \\
\text { Non-ischaemic, } \mathrm{n}(\%)\end{array}$ & $\begin{array}{l}13(22) \\
7(12)\end{array}$ & $\begin{array}{l}0(0) \\
5(17)\end{array}$ & $\begin{array}{l}13(43) \\
2(7)\end{array}$ & $<0.001$ \\
\hline Native T1, ms & $1020 \pm 28$ & $1018 \pm 28$ & $1022 \pm 29$ & 0.594 \\
\hline Extracellular volume, \% & $27.9 \pm 2.9$ & $27.7 \pm 2.7$ & $28.1 \pm 3.1$ & 0.657 \\
\hline \multicolumn{5}{|l|}{ CMR parameters at stress } \\
\hline Cardiac index, $\mathrm{L} / \mathrm{min} / \mathrm{m}^{2}$ & $6.2 \pm 1.2$ & $6.7 \pm 1.3$ & $5.8 \pm 1.0$ & 0.003 \\
\hline Peak ${ }_{C 1}$, percentile & $8[3,19]$ & $18[7,22]$ & $5[2,8]$ & $<0.001$ \\
\hline Change in cardiac index, $\%$ & $104[72,140]$ & $110[89,141]$ & $87[64,145]$ & 0.255 \\
\hline Change in indexed SV, \% & $26[16,43]$ & $29[18,42]$ & $23[14,48]$ & 0.706 \\
\hline Change in LVEF, \% & $21[13,36]$ & $28[16,39]$ & $19[10,36]$ & 0.119 \\
\hline Perfusion defects, n (\%) & $18(30)$ & $2(7)$ & $16(64)$ & $<0.001$ \\
\hline Regional wall motion abnormalities, n (\%) & $30(50)$ & $3(10)$ & $27(90)$ & $<0.001$ \\
\hline
\end{tabular}


Table 1 (continued)

ACE Angiotensin-converting-enzyme, ARB Angiotensin II receptor blockers, BMI Body mass index, BSA Body surface area, CAD coronary artery disease, EDV Enddiastolic volume, ESV End-systolic volume, FFR Fractional flow reserve, $L V$ Left ventricular, LVEF left ventricular ejection fraction, Peak ${ }_{C I}$ Peak exercise cardiac index percentile-rank, RV Right ventricular, SV Stroke volume

Table 2 Univariate and multivariate analysis of ExCMR parameters at rest and stress

\begin{tabular}{|c|c|c|c|c|}
\hline & \multicolumn{2}{|l|}{ Univariate } & \multicolumn{2}{|l|}{ Multivariate } \\
\hline & OR $[95 \% \mathrm{Cl}]$ & $P$ value & OR $[95 \% \mathrm{Cl}]$ & $P$ value \\
\hline LVEF & $0.94[0.88-1.00]$ & 0.058 & - & - \\
\hline RWMA at rest & 16.18 [0.69-378.80] & 0.084 & - & - \\
\hline Late gadolinium enhancement & $7.76[1.98-30.42]$ & 0.003 & $6.15[0.74-51.37]$ & 0.030 \\
\hline Peak $_{\mathrm{Cl}}$ & $0.90[0.83-0.96]$ & 0.003 & $0.93[0.84-1.03]$ & 0.079 \\
\hline Change in cardiac index & $1.00[0.99-1.01]$ & 0.355 & - & - \\
\hline Change in SVi & $1.00[0.98-1.02]$ & 0.869 & - & - \\
\hline Change in LVEF & $0.98[0.95-1.01]$ & 0.167 & - & - \\
\hline RWMA & $61.74[12.48-305.51]$ & $<0.001$ & $22.90[3.09-169.52]$ & 0.010 \\
\hline Perfusion defects & $34.19[7.16-163.37]$ & $<0.001$ & $6.77[0.81-56.66]$ & 0.020 \\
\hline
\end{tabular}

CI Confidence Interval, LVEF Left ventricular ejection fraction, OR Odd ratio, Peak ${ }_{C I}$ Peak exercise cardiac index percentile-rank, $R W M A$ Regional wall motion abnormalities, SVi Stroke volume index
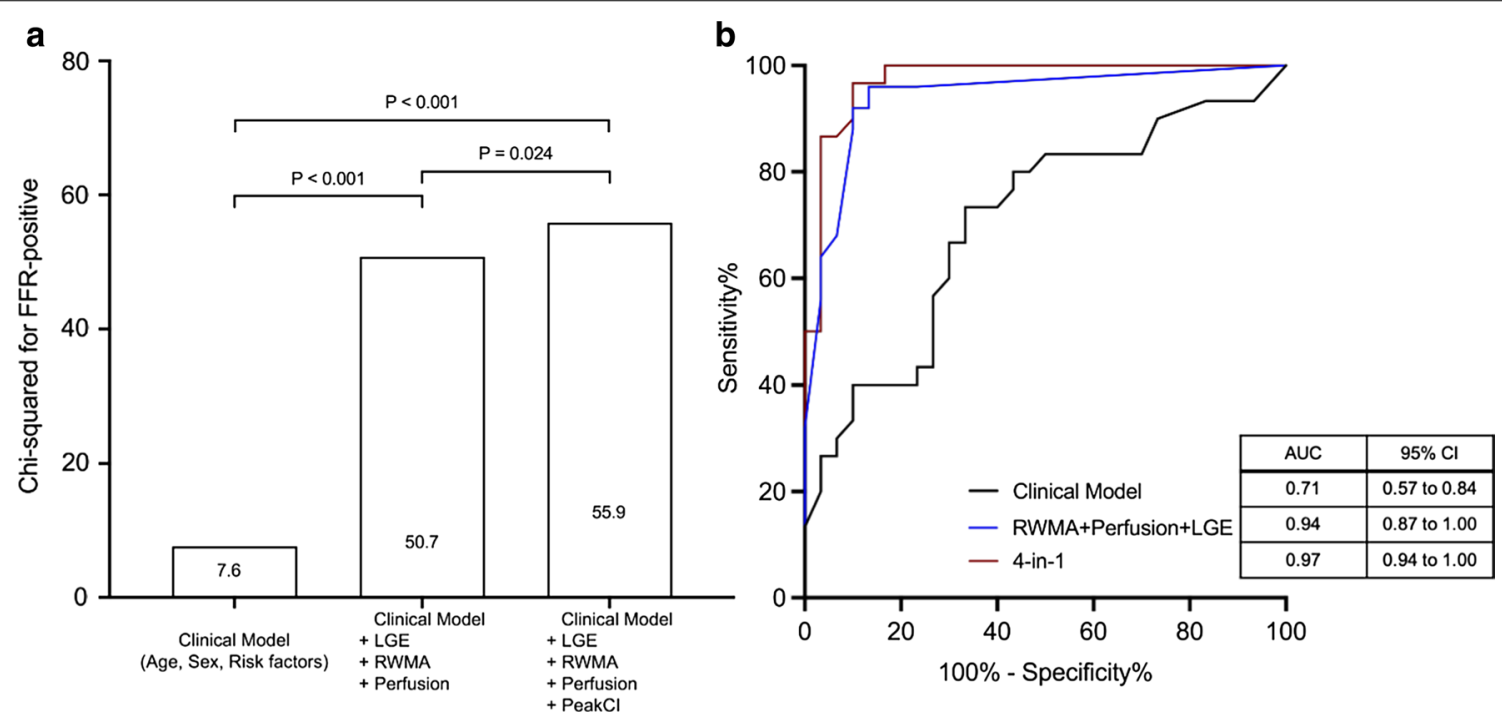

Fig. 2 Test Performance of Exercise CMR. a Change in global $x^{2}$ value of the multiparametric "4-in-1" approach consisting of inducible regional wall motion abnormalities (RWMA), perfusion defects, late gadolinium enhancement (LGE) and peak exercise cardiac index (Peak $\mathrm{C}_{\mathrm{C}}$ ). The clinical model consisted of age, sex and coronary risk factors (diabetes, hypertension, dyslipidaemia and smoking history). b Receiver operating characteristic curves to compare the discriminative abilities of the different models. A multiparametric model consisting of inducible RWMA, perfusion defects, LGE and Peak $\mathrm{Cl}_{\mathrm{C}}$ offered the highest discrimination between those with and without significant coronary artery disease confirmed on fractional flow reserve

increase the sensitivity to detect RWMA. With treadmill exercise CMR, there is a likelihood of reduced sensitivity because of the small delay in transferring the patients to the scanner $[29,30]$. Instead of relying on one parameter, the novel ExCMR protocol combines different diagnostic strengths of regional wall motion abnormalities, perfusion defects, LGE and exercise capacity in a single stress modality to improve diagnostic confidence in detecting significant CAD. The promising results obtained from ExCMR protocol could potentially alter the paradigm of ischaemia evaluation. It is noteworthy to highlight that subsequent 


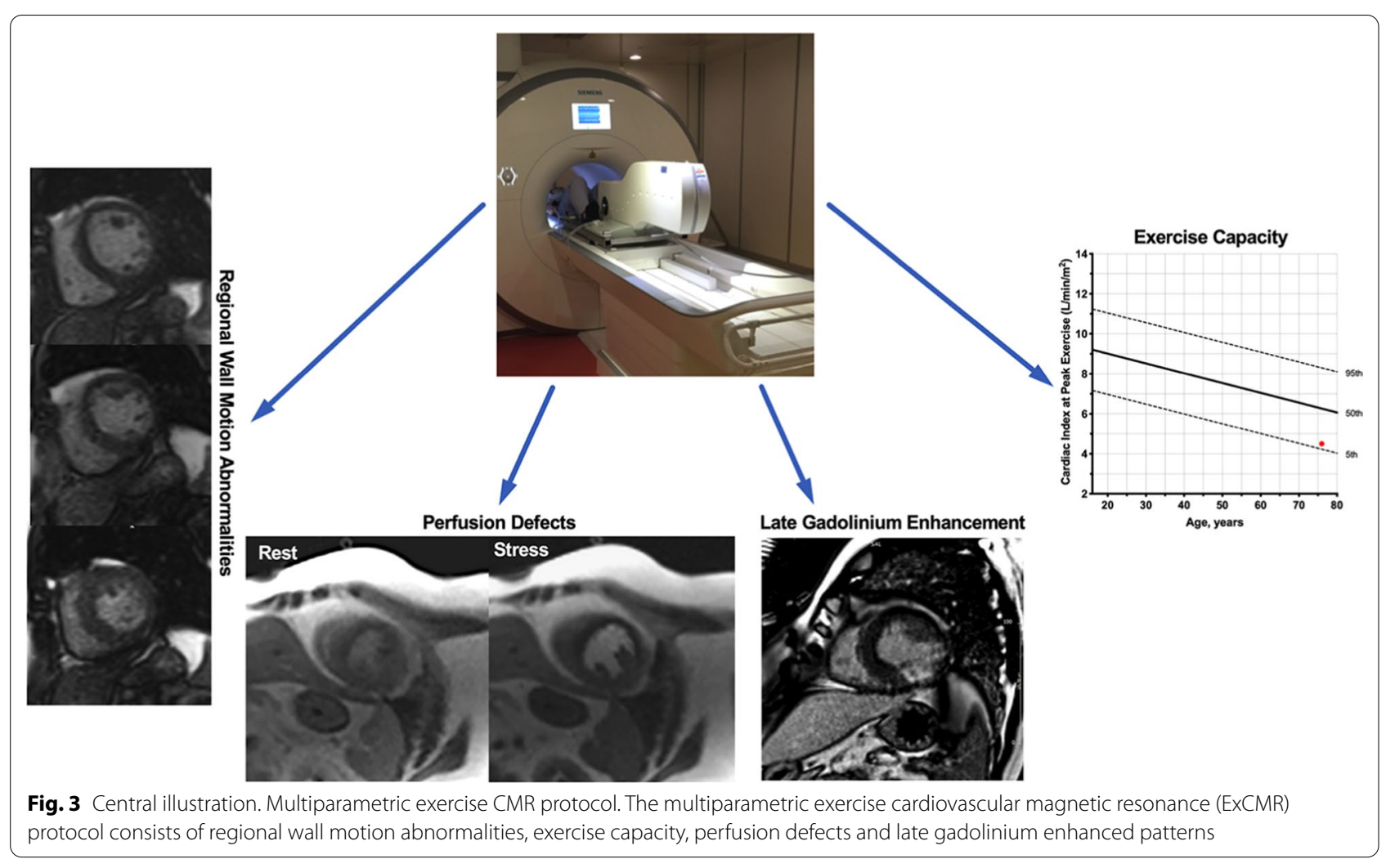

management (initial medical therapy or invasive strategy) will need to be tailored to the individual patient, particularly in the background of recent ISCHEMIA trial [31].

In our study, FFR-positive patients had reduced exercise capacity assessed using peak cardiac index, with a stepwise decrease in Peak ${ }_{\mathrm{CI}}$ according to severity of disease. This observation is consistent with findings from studies in exercise radionuclide angiography and cardiopulmonary tests [32, 33]. Unlike peak LVEF and other exercise parameters, Peak $\mathrm{CI}_{\mathrm{CI}}$ is the only stress measure that incorporates a physiologic parameter (HR) in response to exercise stress [14]. At low exercise levels, SV and HR increase linearly with increased work rate. At higher exercise levels, oxygen supply-demand imbalance in the myocardial regions supplied by the stenotic vessels cause regional dysfunction and consequently, decreased SV. As a physiological response, HR increases to compensate for the decreased SV in order to maintain adequate cardiac output [34]. In patients with advanced CAD, chronotropic incompetence impedes adequate HR response to further increase cardiac output [35]. Although temporal and spatial resolutions are lower in real-time cine images compared to traditional segmented-bSSFP images, we have demonstrated in a previous study that excellent agreement between volumetric quantification between the two image acquisitions can be achieved [14]. Accurate measurement of SV and cardiac index at stress is a strength unparallel in other non-invasive imaging modalities. However, other cardiac conditions such as microvascular disease or cardiomyopathies without epicardial obstructive CAD may be associated with similar poor exercise response $[15,36]$. Thus, $\mathrm{Peak}_{\mathrm{CI}}$ cannot be used as a single measure of significant CAD.

Previous studies have demonstrated diagnostic and prognostic value of exercise capacity in patients with CAD [33, 37, 38]. Our study supports these observations. Peak $_{\mathrm{CI}}$ offered a small but significant incremental value over stress CMR parameters (inducible RWMA and perfusion) and LGE in diagnosing significant CAD. The significance of this finding will need to be confirmed in larger cohorts of patients with CAD.

The maximum HR achieved with ExCMR is of topical interest. The recommended HR criteria of $85 \%$ APMHR to define adequate stress was based on treadmill exercise [39]. As the haemodynamic responses to the different stress modalities are different, we expect the maximum HR achieved with supine cycling to be lower than upright cycling and treadmill [40, 41]. In this study, the patients with suspected CAD achieved $76 \pm 11 \%$ APMHR compared with $83 \pm 3 \%$ and $78 \pm 7 \%$ APMHR in healthy subjects and athletes, respectively [15]. Despite achieving 
HRs lower than the recommended 85\% APMHR, we have demonstrated similar diagnostic performance in patients stratified by median APMHR. There are postulates that supine exercise increases myocardial oxygen demand and the increased LV filling pressure in the supine position may decrease coronary perfusion gradient during diastole, precipitating myocardial ischaemia [41, 42]. These findings may suggest APMHR is not the only consideration when assessing the adequacy of exercise stress CMR.

\section{Study limitations}

Free-breathing exercise stress perfusion image acquisition at high $\mathrm{HR}$ remains challenging to achieve optimal spatial and temporal resolution due to breathing and cardiac motion. This would affect stress perfusion image quality and results in difficulty in the interpretation of images and lower interobserver agreement of perfusion assessment as compared to RWMA and LGE. Newer imaging sequences such as simultaneous multi-slice [43] or compressed sensing $[44,45]$ will likely provide better spatial coverage whilst shorten readout duration to minimize motion. Our ExCMR imaging protocol was designed and conducted on $1.5 \mathrm{~T}$ scanner. $3 \mathrm{~T}$ systems are now widely available and offer higher signal-to-noise and contrast-to-noise ratios that may have theoretical strengths of improved diagnostic accuracy for perfusion imaging. This warrants future validation. This is a single center experience in patients with intermediate pre-test CAD risk and relatively high CAD prevalence. Further larger, multi-center studies are needed to confirm our findings in lower risk cohorts. However, a single center is also essential at this point to ensure strict adherence to imaging and FFR protocols, crucial standards to establish for a first study.

\section{Conclusion}

ExCMR using supine cycle ergometer demonstrates feasibility in assessing multiple CMR parameters that in combination, have high accuracy in diagnosing significant CAD as defined by invasive FFR. Future studies are now needed to validate these findings.

\section{Supplementary Information}

The online version contains supplementary material available at https://doi. org/10.1186/s12968-021-00705-8.

Additional file 1. Online Supplementary.

\section{Abbreviations}

APMHR: Age-predicted maximal heart rate; AUC: Area under the curve; bSSFP: Balanced steady state free precession; CAD: Coronary artery disease; Cl: Confidence Interval; CMR: Cardiovascular magnetic resonance; ECG: Electrocardiogram; ECV: Extracellular volume fraction; ESV: End-systolic volume; ExCMR: Exercise stress CMR; FFR: Fractional flow reserve; HR: Heart rate; LGE:
Late gadolinium enhancement; LV: Left ventricle/left ventricular; LVEF: Left ventricular ejection fraction; MOLLI: Modified Look-Locker inversion recovery; Peak $_{\mathrm{cl}}$ : Peak exercise cardiac index percentile-rank; PSIR: Phase sensitive inversion recovery; RWMA: Regional wall motion abnormalities; SV: Stroke volume; SVI: Stroke volume index; $\mathrm{VO}_{2}$ max: Maximal oxygen uptake.

\section{Acknowledgements}

We thank the radiographers at the Department of Cardiovascular Magnetic Resonance Imaging, National Heart Centre Singapore, the nurses and medical technologists at the Cardiac Catheterisation Laboratory, National Heart Centre Singapore for their help in the study. We are grateful for the participation of all study participants.

\section{Authors' contributions}

TTL conceived the study, formulated the hypothesis, analysed the data and prepared the manuscript. BWYA collected and analysed the data. JAB optimised the CMR sequences and performed scans for the participants. CYC, KKY, PEHW, KWH, JWCT performed invasive coronary angiogram and FFR procedures. PTL recruited the patients, analysed the CMR data and provided clinical insights in the study. CWLC and SAC supervised the study and edited the manuscript. All authors read and approved the final manuscript.

\section{Funding}

The study is supported by grants from the National Medical Research Foundation Singapore (NMRC/OFYIRG/030/2017 and NMRC/CGAug 16/M006).

\section{Availability of data and materials}

The datasets used and analysed during the current study are available from the corresponding author on reasonable request.

\section{Ethics approval and consent to participate}

The study was conducted in accordance with the Declaration of Helsinki and approved by the Singhealth Centralised Institutional Review Board (2017/2123). Written informed consent was obtained from all individuals.

\section{Consent of publication}

Not applicable.

\section{Competing interests}

The authors declare that they have no competing interests.

\section{Author details}

${ }^{1}$ National Heart Research Institute Singapore, National Heart Centre Singapore, 5 Hospital Drive, Singapore 169609, Singapore. ${ }^{2}$ Cardiovascular Sciences ACP, Duke-NUS Graduate Medical School, Singapore, Singapore. ${ }^{3}$ Department of Cardiology, National Heart Centre Singapore, Singapore, Singapore.

${ }^{4}$ National Heart and Lung Institute, Imperial College, London, UK.

Received: 21 July 2020 Accepted: 6 January 2021

Published online: 04 March 2021

\section{References}

1. Greenwood JP, Maredia N, Younger JF, Brown JM, Nixon J, Everett CC, Bijsterveld P, Ridgway JP, Radjenovic A, Dickinson CJ, et al. Cardiovascular magnetic resonance and single-photon emission computed tomography for diagnosis of coronary heart disease (CE-MARC): a prospective trial. Lancet. 2012;379(9814):453-60.

2. Schwitter J, Wacker CM, Wilke N, Al-Saadi N, Sauer E, Huettle K, Schonberg SO, Luchner A, Strohm O, Ahlstrom H, et al. MR-IMPACT II: Magnetic Resonance Imaging for Myocardial Perfusion Assessment in Coronary artery disease Trial: perfusion-cardiac magnetic resonance vs. singlephoton emission computed tomography for the detection of coronary artery disease: a comparative multicentre, multivendor trial. Eur Heart $\mathrm{J}$. 2013;34(10):775-81

3. Takx RA, Blomberg BA, El Aidi H, Habets J, de Jong PA, Nagel E, Hoffmann $U$, Leiner T. Diagnostic accuracy of stress myocardial perfusion imaging compared to invasive coronary angiography with fractional flow reserve meta-analysis. Circ Cardiovasc Imaging. 2015;8(1):e002666. 
4. Bingham SE, Hachamovitch R. Incremental prognostic significance of combined cardiac magnetic resonance imaging, adenosine stress perfusion, delayed enhancement, and left ventricular function over preimaging information for the prediction of adverse events. Circulation. 2011;123(14):1509-18.

5. Lipinski MJ, McVey CM, Berger JS, Kramer CM, Salerno M. Prognostic value of stress cardiac magnetic resonance imaging in patients with known or suspected coronary artery disease: a systematic review and meta-analysis. J Am Coll Cardiol. 2013;62(9):826-38.

6. Heitner JF, Kim RJ, Kim HW, Klem I, Shah DJ, Debs D, Farzaneh-Far A, Polsani V, Kim J, Weinsaft J, et al. Prognostic value of vasodilator stress cardiac magnetic resonance imaging: a multicenter study with 48000 patient-years of follow-up. JAMA Cardiol. 2019;4(3):256-64.

7. Nagel E, Greenwood JP, McCann GP, Bettencourt N, Shah AM, Hussain ST, Perera D, Plein S, Bucciarelli-Ducci C, Paul M, et al. Magnetic resonance perfusion or fractional flow reserve in coronary disease. $N$ Engl I Med. 2019;380(25):2418-28.

8. Kwong RY, Ge Y, Steel K, Bingham S, Abdullah S, Fujikura K, Wang W, Pandya A, Chen Y-Y, Mikolich JR, et al. Cardiac magnetic resonance stress perfusion imaging for evaluation of patients with chest pain. J Am Coll of Cardiol. 2019;74(14):1741-55.

9. Lancellotti P, Pellikka PA, Budts W, Chaudhry FA, Donal E, Dulgheru R, Edvardsen T, Garbi M, Ha JW, Kane GC, et al. The clinical use of stress echocardiography in non-ischaemic heart disease: recommendations from the european association of cardiovascular imaging and the American Society of Echocardiography. J Am Soc Echocardiogr. 2017;30(2):101-38.

10. Varga A, Garcia MA, Picano E. International stress echo complication R: safety of stress echocardiography (from the International Stress Echo Complication Registry). Am J Cardiol. 2006;98(4):541-3.

11. Kramer CM, Barkhausen J, Flamm SD, Kim RJ, Nagel E. Society for cardiovascular magnetic resonance board of trustees task force on standardized P: standardized cardiovascular magnetic resonance (CMR) protocols 2013 update. J Cardiovasc Magn Reson. 2013;15:91.

12. Le TT, Huang W, Bryant JA, Cook SA, Chin CW. Stress cardiovascular magnetic resonance imaging: current and future perspectives. Expert Rev Cardiovasc Ther. 2017;15(3):181-9.

13. Raman SV, Dickerson JA, Mazur W, Wong TC, Schelbert EB, Min JK, Scandling D, Bartone C, Craft JT, Thavendiranathan P, et al. Diagnostic performance of treadmill exercise cardiac magnetic resonance: the prospective, multicenter Exercise CMR's Accuracy for Cardiovascular Stress Testing (EXACT) trial. J Am Heart Assoc. 2016. https://doi.org/10.1161/ JAHA.116.003811.

14. Le TT, Bryant JA, Ting AE, Ho PY, Su B, Teo RC, Gan JS, Chung YC, O'Regan DP, Cook SA, et al. Assessing exercise cardiac reserve using real-time cardiovascular magnetic resonance. J Cardiovasc Magn Reson. 2017;19(1):7.

15. Le T-T, Bryant JA, Ang BWY, Pua CJ, Su B, Ho PY, Lim S, Huang W, Lee PT, Tang HC, et al. The application of exercise stress cardiovascular magnetic resonance in patients with suspected dilated cardiomyopathy. J Cardiovasc Magn Reson. 2020;22(1):10.

16. Campeau L. Letter: Grading of angina pectoris. Circulation. 1976;54(3):522-3.

17. Xue H, Zuehlsdorff S, Kellman P, Arai A, Nielles-Vallespin S, Chefdhotel C, Lorenz CH, Guehring J. Unsupervised inline analysis of cardiac perfusion MRI. Med Image Comput Comput Assist Interv. 2009;12(Pt 2):741-9.

18. Rajiah P, Desai MY, Kwon D, Flamm SD. MR imaging of myocardial infarction. RadioGraphics. 2013;33(5):1383-412.

19. Kim RJ, Wu E, Rafael A, Chen EL, Parker MA, Simonetti O, Klocke FJ, Bonow RO, Judd RM. The use of contrast-enhanced magnetic resonance imaging to identify reversible myocardial dysfunction. N Engl J Med. 2000;343(20):1445-53.

20. Chin CW, Semple S, Malley T, White AC, Mirsadraee S, Weale PJ, Prasad S, Newby DE, Dweck MR. Optimization and comparison of myocardial T1 techniques at $3 \mathrm{~T}$ in patients with aortic stenosis. Eur Heart J Cardiovasc Imaging. 2014;15(5):556-65.

21. Messroghli DR, Moon JC, Ferreira VM, Grosse-Wortmann L, He T, Kellman P, Mascherbauer J, Nezafat R, Salerno M, Schelbert EB, et al. Clinical recommendations for cardiovascular magnetic resonance mapping of T1, T2, T2* and extracellular volume: a consensus statement by the Society for Cardiovascular Magnetic Resonance (SCMR) endorsed by the European
Association for Cardiovascular Imaging (EACVI). J Cardiovasc Magn Reson. 2017;19(1):75.

22. Liu A, Wijesurendra RS, Francis JM, Robson MD, Neubauer S, Piechnik SK, Ferreira VM. Adenosine stress and rest T1 mapping can differentiate between ischemic, infarcted, remote, and normal myocardium without the need for gadolinium contrast agents. JACC Cardiovasc Imaging. 2016;9(1):27-36.

23. Schulz-Menger J, Bluemke DA, Bremerich J, Flamm SD, Fogel MA, Friedrich MG, Kim RJ, von Knobelsdorff-Brenkenhoff F, Kramer CM, Pennell DJ, et al. Standardized image interpretation and post processing in cardiovascular magnetic resonance: Society for Cardiovascular Magnetic Resonance (SCMR) Board of Trustees Task Force on Standardized Post Processing. J Cardiovasc Magn Reson. 2013;15(1):35.

24. Toth GG, Johnson NP, Jeremias A, Pellicano M, Vranckx P, Fearon WF, Barbato E, Kern MJ, Pijls NH, De Bruyne B. Standardization of fractional flow reserve measurements. J Am Coll Cardiol. 2016;68(7):742-53.

25. Tonino PA, Fearon WF, De Bruyne B, Oldroyd KG, Leesar MA, Ver Lee PN, Maccarthy PA, Van't Veer M, Pijls NH. Angiographic versus functional severity of coronary artery stenoses in the FAME study fractional flow reserve versus angiography in multivessel evaluation. J Am Coll Cardiol. 2010;55(25):2816-21.

26. Bursac Z, Gauss CH, Williams DK, Hosmer DW. Purposeful selection of variables in logistic regression. Source Code Biol Med. 2008;3:17-17.

27. La Gerche A, Claessen G, Van de Bruaene A, Pattyn N, Van Cleemput J, Gewillig M, Bogaert J, Dymarkowski S, Claus P, Heidbuchel H. Cardiac MRl: a new gold standard for ventricular volume quantification during highintensity exercise. Circ Cardiovasc Imaging. 2013;6(2):329-38.

28. Nakamori S, Fahmy A, Jang J, El-Rewaidy H, Neisius U, Berg S, Goddu B, Pierce P, Rodriguez J, Hauser T, et al. Changes in Myocardial Native T1 and T2 after exercise stress: a noncontrast CMR pilot study. JACC CardiovasC Imaging. 2020;13(3):667-80.

29. Fletcher GF, Ades PA, Kligfield P, Arena R, Balady GJ, Bittner VA, Coke LA, Fleg JL, Forman DE, Gerber TC, et al. Exercise standards for testing and training: a scientific statement from the American Heart Association. Circulation. 2013;128(8):873-934.

30. Hecht HS, DeBord L, Sotomayor N, Shaw R, Dunlap R, Ryan C. Supine bicycle stress echocardiography: peak exercise imaging is superior to postexercise imaging. J Am Soc Echocardiogr. 1993;6(3 Pt 1):265-71.

31. Maron DJ, Hochman JS, Reynolds HR, Bangalore S, O'Brien SM, Boden WE, Chaitman BR, Senior R, Lopez-Sendon J, Alexander KP, et al. Initial Invasive or Conservative Strategy for Stable Coronary Disease. N Engl J Med. 2020;382(15):1395-407.

32. Rerych SK, Scholz PM, Newman GE, Sabiston DC, Jones RH. Cardiac function at rest and during exercise in normals and in patients with coronary heart disease: evaluation by radionuclide angiocardiography. Ann Surg. 1978;187(5):449-64.

33. Belardinelli R, Lacalaprice F, Carle F, Minnucci A, Cianci G, Perna G, D'Eusanio G. Exercise-induced myocardial ischaemia detected by cardiopulmonary exercise testing. Eur Heart J. 2003;24(14):1304-13.

34. Chaudhry S, Kumar N, Behbahani H, Bagai A, Singh BK, Menasco N, Lewis GD, Sperling L, Myers J. Abnormal heart-rate response during cardiopulmonary exercise testing identifies cardiac dysfunction in symptomatic patients with non-obstructive coronary artery disease. Int J Cardiol. 2017;228:114-21.

35. Dresing TJ, Blackstone EH, Pashkow FJ, Snader CE, Marwick TH, Lauer MS. Usefulness of impaired chronotropic response to exercise as a predictor of mortality, independent of the severity of coronary artery disease. Am J Cardiol. 2000;86(6):602-9.

36. Bechsgaard DF, Hove JD, Suhrs HE, Bové KB, Shahriari P, Gustafsson I, Prescott E. Women with coronary microvascular dysfunction and no obstructive coronary artery disease have reduced exercise capacity. Int J Cardiol. 2019;293:1-9.

37. D'Andrea A, Severino S, Caso P, Liccardo B, Forni A, Fusco A, Lo Piccolo R, Scherillo M, Mininni N, Calabro R. Prognostic value of supine bicycle exercise stress echocardiography in patients with known or suspected coronary artery disease. Eur J Echocardiogr. 2005;6(4):271-9.

38. Hung RK, Al-Mallah MH, McEvoy JW, Whelton SP, Blumenthal RS, Nasir K, Schairer JR, Brawner C, Alam M, Keteyian SJ, et al. Prognostic value of exercise capacity in patients with coronary artery disease: the FIT (Henry Ford Exerclse Testing) project. Mayo Clin Proc. 2014;89(12):1644-54. 
39. Balady Gary J, Larson Martin G, Vasan Ramachandran S, Leip Eric P, O'Donnell Christopher J, Levy D. Usefulness of exercise testing in the prediction of coronary disease risk among asymptomatic persons as a function of the framingham risk score. Circulation. 2004;110(14):1920-5.

40. Quinn TJ, Smith SW, Vroman NB, Kertzer R, Olney WB. Physiologic responses of cardiac patients to supine, recumbent, and upright cycle ergometry. Arch Phys Med Rehabil. 1995;76(3):257-61.

41. Badruddin SM, Ahmad A, Mickelson J, Abukhalil J, Winters WL, Nagueh SF, Zoghbi WA. Supine bicycle versus post-treadmill exercise echocardiography in the detection of myocardial ischemia: a randomized single-blind crossover trial. J Am Coll Cardiol. 1999;33(6):1485-90.

42. Modesto KM, Rainbird A, Klarich KW, Mahoney DW, Chandrasekaran K, Pellikka PA. Comparison of supine bicycle exercise and treadmill exercise Doppler echocardiography in evaluation of patients with coronary artery disease. Am J Cardiol. 2003;91(10):1245-8.

43. Nazir MS, Neji R, Speier P, Reid F, Stäb D, Schmidt M, Forman C, Razavi R, Plein S, Ismail TF, et al. Simultaneous multi slice (SMS) balanced steady state free precession first-pass myocardial perfusion cardiovascular magnetic resonance with iterative reconstruction at $15 \mathrm{~T}$. J Cardiovasc Magn Reson. 2018;20(1):84.

44. Naresh NK, Haji-Valizadeh H, Aouad PJ, Barrett MJ, Chow K, Ragin AB, Collins JD, Carr JC, Lee DC, Kim D. Accelerated, first-pass cardiac perfusion pulse sequence with radial $k$-space sampling, compressed sensing, and k-space weighted image contrast reconstruction tailored for visual analysis and quantification of myocardial blood flow. Magn Reson Med. 2019;81(4):2632-43

45. Otazo R, Kim D, Axel L, Sodickson DK. Combination of compressed sensing and parallel imaging for highly accelerated first-pass cardiac perfusion MRI. Magn Reson Med. 2010;64(3):767-76.

\section{Publisher's Note}

Springer Nature remains neutral with regard to jurisdictional claims in published maps and institutional affiliations.
Ready to submit your research? Choose BMC and benefit from:

- fast, convenient online submission

- thorough peer review by experienced researchers in your field

- rapid publication on acceptance

- support for research data, including large and complex data types

- gold Open Access which fosters wider collaboration and increased citations

- maximum visibility for your research: over $100 \mathrm{M}$ website views per year

At BMC, research is always in progress.

Learn more biomedcentral.com/submissions 